\title{
Cirugía con circulación extracorpórea e hipotermia en tumores con extensión a vena cava: 20 años de experiencia de la Clínica Universitaria de Navarra
}

\author{
Rioja Zuazu J*, Rodríguez-Rubio Cortadellas FI**, Zudaire Bergera JJ*, Saiz Sansi A*, \\ Rosell Costa D*, Robles García JE*, Rábago G, Berián Polo JM*. \\ *Departamento de Urología, Clínica Universitaria. Universidad de Navarra. Pamplona. \\ **Servicio de Urología, Hospital Universitario Puerto Real, Cádiz. \\ ***Departamento de Cirugía Cardiovascular. Clínica Universitaria. Universidad de Navarra. Pamplona.
}

Actas Urol Esp. 2008;32(4):396-405

\begin{abstract}
RESUMEN
CIRUGÍA CON CIRCULACIÓN EXTRACORPOREA E HIPOTERMIA EN TUMORES CON EXTENSIÓN A VENA CAVA: 20 AÑOS DE EXPERIENCIA DE LA CLÍNICA UNIVERSITARIA DE NAVARRA

Objetivo: Presentamos 20 años de experiencia en pacientes con tumores con extensión a vena cava en los que se realizaron circulación extracorpórea, hipotermia, parada cardiaca y exanguinotransfusión (CEC-H-PC-E) para que junto con la resección tumoral se resecara el trombo tumoral en su totalidad.

Material y Métodos: Entre los años 1985 y 2005 se trataron 28 tumores retroperitoneales: 25 tumores renales, un tumor de Wilms, un rabdomiosarcoma paratesticular y un feocromocitoma. Todos ellos presentaban extensión en forma de trombo en la vena cava por encima de las venas suprahepáticas. A todos se les realizó CEC-H-PC-E para la extracción del trombo tumoral. Se realiza una descripción de la serie así como un análisis de la supervivencia de Kaplan-Meier.

Resultados: Se presentaron complicaciones quirúrgicas en 10 pacientes (35\%), con una mortalidad quirúrgica de dos pacientes (7\%): intraoperatoria por embolismo pulmonar masivo en un paciente y al $4^{\circ}$ día postquirúrgico por embolismo pulmonar. La supervivencia actuarial global fue de $29,1 \pm 10 \%$ a tres años y $17,5 \pm 8 \%$ a cinco años. Analizando por separado aquellos que tienen que no presentan lesiones metastásica ni ganglionares en el diagnóstico su supervivencia fue a tres años del 50,9 $916,3 \%$ y del $38,2 \pm 16 \%$ a cinco años. Mientras aquellos que presentan algún tipo de lesión a distancia presentaron una supervivencia a tres y cinco años de $20,8 \pm 12 \%$ y $10,4 \pm 9 \%$ respectivamente.

Conclusiones: La utilización de técnicas quirúrgicas con CEC-H-PC-E en patologías tumorales que se asocian a trombo en vena cava, esta justificada y su utilización no empeora la supervivencia; está indicada por sus resultados, permitiendo una resección tumoral de una manera segura y reproductible.
\end{abstract}

Palabras clave: Tumor renal. Trombo en cava. Circulación extracorpórea.

\begin{abstract}
EXTRACORPOREAL CIRCULATION AND HYPOTHERMY SURGERY IN TUMORS WITH VENA CAVA EXTENSION: 20 YEARS EXPERIENCE AT THE UNIVERSITY CLINIC OF NAVARRA

Objective: We present our 20 years experience treating patients with vena cava extension in whom an extracorporeal circulation, hypothermia, cardio circulatory arrest (ECC-H-CCA) in order to perform, together with a tumoral resection, a thrombus resection.

Material and Methods: From 1985 to 2005 a total of 28 retroperitoneal tumor were treated: 25 renal cancers, a Wilms tumor, a paratesticular rabdomiosarcoma, and a pheocromocitoma. All of them had an extension by means of thrombus above the suprahepatics veins. All of them were treated by means of ECC-H-CCA for thrombus extraction. A descriptive study of the serie is performed as well as a Kaplan Meyer survival study.

Results: Surgical complications were present within 10 patients (35\%), with a surgical mortality of two patients (7\%): one intra-operatively because a massive embolism of the lungs and the other because of a lung embolism on the $4^{\text {th }}$ post-operative day. Global actuarial survival was $29.1 \pm 10 \%$ at three years and $17,5 \pm 8 \%$ at five years. Analyzing only who do not have metastatic lesions, nor lymph nodes at diagnosis their three year survival was $50,9 \pm 16,3 \%$ and $32,2 \pm 16 \%$ at five years. Mean while those who have any metastatic lesion at diagnosis their three and five years survival was $20,8 \pm 12 \%$ and $10,4 \pm 9 \%$ respectively.

Conclusions: The employ of surgical techniques with ECC-H-CCA with in oncological pathology associated with vena cava thrombus is justified and its employment does not worsen the survival; it is indicated because its results, allowing a complete tumoral resection in a safe and reproducible fashion.
\end{abstract}

Keywords: Renal tumor. Cava thrombus. Extracorporeal circulation. 
$\mathrm{U}$ na de las características del tumor renal es su diseminación a través del sistema venoso con formación de trombos venosos tumorales que progresan a través de la cava inferior y que pueden llegar en ocasiones a la aurícula derecha ${ }^{1}$. En el momento del diagnóstico podemos encontrar invasión de la vena renal en el 20-35\% de los casos, mientras que su extensión en la vena cava inferior ocurre en un 4-10\% de los pacientes y en un 10$25 \%$ de estos, el trombo se extiende por encima de las venas suprahepáticas hasta la aurícula derecha o incluso el ventrículo derecho ${ }^{2,3}$.

Los avances en las técnicas quirúrgicas y en los cuidados perioperatorios que se han producido en las últimas tres décadas, han permitido que, incluso los tumores con extensión en la aurícula derecha, puedan ser resecados con relativa seguridad 4 . En este sentido la nefrectomía con trombectomía es el tratamiento de elección en estos casos $^{5}$. La resección del trombo en cava y aurícula, requiere la colaboración de un equipo de cirugía cardiaca, para la realización de técnicas de by-pass veno-venoso o cardiopulmonar con o sin exanguinotransfusión. A causa de su mortalidad que varía entre un 10 y un 50\% en distintas series publicadas $^{2,6,7}$, este tipo de cirugías debe realizarse en centros experimentados y con fácil coordinación entre disciplinas. Por otro lado, la literatura transmite que la existencia de un trombo tumoral, por sí misma, no está asociada con un peor pronóstico de la enfermedad y los pacientes sin metástasis pueden alcanzar supervivencias largas después de la resección del mismo ${ }^{4,8}$.

Otros tumores, algunos de clara implicación del urólogo, también se han asociado a la presencia de trombo tumoral en vena cava: carcinoma adrenal, carcinoma de pelvis renal, leiomiosarcoma de cava, sarcoma de partes blandas, hepatocarcinoma, tumor de ovario y testículo, feocromocitoma y tumor de Wilms ${ }^{9-13}$.

Presentamos la actualización de los 20 años de experiencia quirúrgica de una única institución en pacientes con tumor y extensión en trombo en cava, que alcanzaban como mínimo las venas suprahepáticas o hasta aurícula, en los que se realizó circulación extracorpórea, hipotermia, parada cardiaca y exanguinotransfusión (CEC-H-PC-E) ${ }^{14-20}$.

\section{MATERIAL Y MÉTODOS}

En 1985, un año después de que Marshall ${ }^{21}$ describiese la aplicación de CEC-H-PC-E en el trata- miento quirúrgico del tumor renal con trombo en cava, se realizó el primer caso en la Clínica Universitaria de Navarra (CUN) de tumor retroperitoneal con trombectomía empleando esta técnica y desde entonces se han realizado 28 procedimientos. Los 28 casos se distribuían en 25 tumores renales (estadios TNM 2002 T3b/c); un tumor de Wilms; un rabdomiosarcoma paratesticular y un feocromocitoma.

\section{Protocolo de estadificación}

A todos los pacientes intervenidos, se les realizo una estadificación loco-regional y a distancia mediante tomografía computerizada (TC) y/o resonancia magnética, gammagrafía ósea y resonancia cerebral en caso de presentar algún tipo de sintomatología neurológica, así como ecografía-doppler prequirúrgica para establecer correctamente la altura del trombo. En los casos iniciales de la serie (1985) se realizaba cavografía como técnica de elección para determinar la altura de trombo en vena cava.

Para la estadificación del trombo tumoral, se utilizó una modificación de la clasificación de Neves y Zinke $^{22}$ y del $\mathrm{TNM}^{23}$, la clasificación de la CUN que nos permitió una planificación de la estrategia quirúrgica a seguir, en la cual el punto de referencia son las venas suprahepáticas. Los trombos tumorales quedaron divididos en tres tipos, B1 cuando el trombo no alcanza las venas suprahepáticas, B2 cuando está a la altura de las mismas, y C, cuando las supera (Fig. 1). En función de esta clasificación se planifica la cirugía, con o sin cirugía extracorpórea, de manera que a los pacientes con un estadio

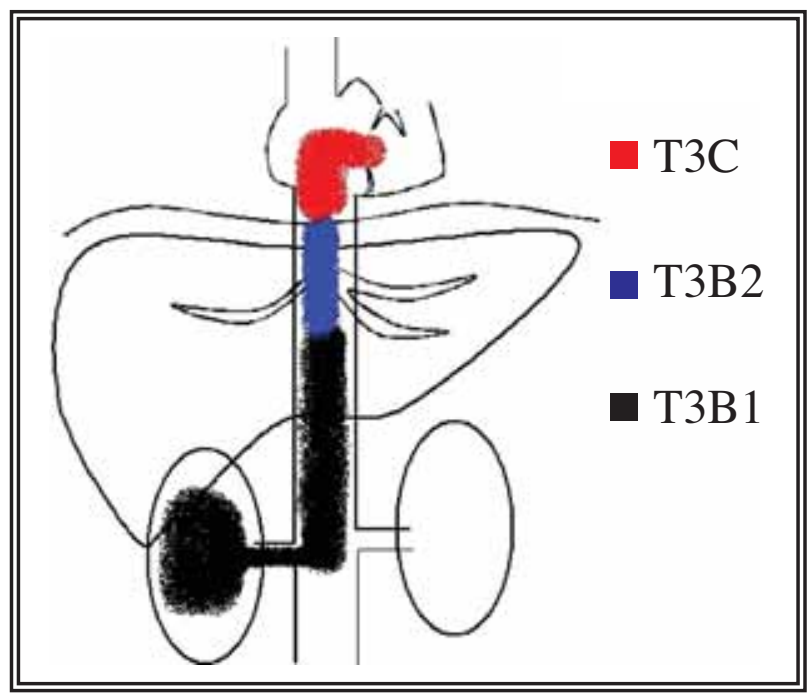

FIGURA 1. Clasificación CUJ para el trombo tumoral. 
B1 se realiza nefrectomía ampliada con clampaje selectivo de la vena cava y trombectomía, mientras que si presentan un estadio $\mathrm{B} 2$ o $\mathrm{C}$ se realiza la cirugía abdominal más CEC-H-PC-E. Los 28 pacientes estudiados en este trabajo fueron operados con esta última técnica al ser todos B2 o C.

\section{Estadio de los 25 tumores renales}

Siguiendo nuestro sistema de clasificación del trombo, la serie de pacientes se distribuía de la siguiente manera: 12 pacientes eran tipo B2 (48\%) y otros 13 eran tipo C (52\%). Respecto a la estadificación ganglionar y metastática, 16 pacientes eran NO (64\%) y 18 pacientes (72\%) no presentaban metástasis a distancia (MO).

\section{Estadio del tumor de Tumor de Wilms}

Niño de 15 años con un tumor de Wilms derecho estadio III, que presentaba infiltración de la vena cava (clasificación de la CUN T3B2).

\section{Estadio del rabdomiosarcoma paratesticular}

Niño de 13 años con previa orquiectomía inguinal (rabdomiosarcoma partesticular embrionario) y posterior quimioterapia y laparotomía exploradora sin evidencias de afectación retroperitoneal. A los 16 meses un control con TC revela una masa retroperitoneal que engloba la vena cava inferior extendiéndose a su luz y alcanzando el nivel de las venas suprahepáticas (clasificación CUN del trombo T3B2).

\section{Estadio del feocromocitoma}

Mujer de 45 años que es derivada a nuestro centro presentando un cuadro de fracaso multiorgánico secundario a síndrome de Budd-Chiari por feocromocitoma derecho (4 cm. de diámetro) con un trombo tumoral en la vena cava que ascendía hasta la altura de las venas suprahepáticas (clasificación CUN del tromboT3B2) provocando trombosis de las mismas que ocasionaba necrosis de los segmento 6 y 7 del lóbulo hepático derecho. El trombo en cava se extendía distalmente hasta la bifurcación de la venas ilíacas en forma de trombo plaquetario secundario $^{18}$ (Fig. 1).

\section{Técnica quirúrgica}

Se realiza habitualmente incisión subcostal bilateral (tipo chevron) combinada con una esternoto- mía media, la cual permite una exposición suficiente del campo operatorio. Se realiza exposición del retroperitoneo, con maniobra de Kocher en el lado derecho. En ocasiones resulta útil liberar el hígado mediante volteo del mismo, para exponer la vena cava abdominal en toda su extensión. Posteriormente se realiza disección de la masa retroperitoneal, intentando un control arterial (renal en caso de tumor renal) lo más precoz posible, permitiendo un mejor control hemostático. La disección debe ser muy cuidadosa por la presencia de abundante circulación colateral, que tiene tendencia al sangrado. El equipo de cirugía cardiaca inicia el by-pass cardio-pulmonar cuando se ha conseguido la movilización completa de la lesión tumoral y pendiente únicamente del componente venoso junto con una hemostasia exquisita. Estas dos condiciones son claves e inexcusables. La heparinización es necesaria y por ello la hemostasia previa y el control arterial de la masa. Por otro lado, como el tiempo de bypass y de disección del trombo intravenosos e intraauricular y posterior reconstrucción no debe exceder los 45 minutos, se debe tener la masa lo más delimitada posible.

Para iniciar el by-pass, se realiza la pericardiotomía, y tanto la aorta como la aurícula derecha son canuladas para iniciar la circulación extracorpórea. Se pasa a inducir la hipotermia y cuando el corazón empieza a fibrilar se realiza el clampaje de la aorta y se perfunde a nivel aórtico (seno coronario) la solución cardiopléjica. En este momento el paciente se encuentra en circulación extracorpórea con parada cardiaca y se sigue induciendo la hipotermia hasta alcanzar una temperatura rectal o vesical (y no esofágica) de $14^{\circ} \mathrm{C}$. Alcanzada esa temperatura se produce la exanguinación, clampando la cánula arterial y depositando toda la sangre en el oxigenador y en el reservorio. Una vez realizada la exanguinación, se para la bomba de circulación extracorpórea, pudiendo realizarse la cavotomía y auricolotomía, en el caso de los T3C, sobre un lecho quirúrgico exangüe. Se realiza la disección del trombo y se extrae todo en una pieza (tumor y trombo) por el campo abdominal. En ocasiones y debido al tamaño del trombo (casos T3C) es preciso extraer parte del trombo a través de la auriculotomía. La vena cava, en excepcionales ocasiones presenta infiltración tumoral, que requiera resección de la misma, por lo general, el tumor presenta una capa de fibrina que 
la adhiere al endotelio pero que habitualmente resulta de fácil disección. La sutura de la cavotomía debe permitir una luz venosa suficiente para el drenaje del riñón contralateral, es por esto por lo que en ocasiones se requieren parche de pericardio o de Gore-tex $^{\circledR}$. El tiempo que se dispone para realizar todos estos pasos no puede sobrepasar los $45 \mathrm{minu}-$ tos, ya que a partir de ese momento el riesgo daño cerebral inducido por la anoxia aumenta, así como la acidosis metabólica, por lo que es necesario monitorizar el $\mathrm{pH}$ arterial.

Una vez concluidos todos estos pasos, se reanuda el by-pass cardiopulmonar y se calienta progresivamente al paciente. Habitualmente, el corazón se reinicia con ritmo sinusal de manera espontánea, pero en ocasiones lo hace con fibrilación ventricular siendo necesario la utilización de un desfibrilador. Una vez que el corazón se encuentra en ritmo sinusal, el paciente se encuentra por encima de los $35^{\circ} \mathrm{C}$ a nivel rectal o vesical y con los marcadores cardíacos temporales implantados se puede salir de bomba de circulación extracorpórea. Una vez finalizado todos estos pasos se procede al cierre de la laparotomía y de la toracotomía previa colocación de los drenajes oportunos.

La medición de la temperatura transrectal es debido al artefacto que provoca la medición transesofágica, pues está en contacto con la aorta que es por donde se infunde la hipotermia y el calentamiento.

A todos ellos los tumores renales, incluido el Wilms, se les realizo una nefrectomía con trombectomía previa CEC-H-PC-E para la extracción del trombo. En el caso del rabdomiosarcoma partesticular se realizó linfadenectomía retroperitoneal más CEC-H-PC-E y extracción de la totalidad del trombo y comprobación de origen del trombo tumoral en la vena gonadal.

En el caso feocromocitoma, se realizó una hepatectomía anterior derecha, con maniobra de Pringle intermitente, sin voltear el hígado, seccionando el hígado hasta llegar a cara anterior de la cava retrohepática. Disección de vena cava desde iliacas primitivas hasta vena renal derecha. Liberación de la glándula suprarrenal derecha y exéresis de suprarrenal e hígado derecho a falta de cavotomía. La CEC-H-PC-E permitió la cavotomía inferior a nivel de suprarrenal y aurículotomía derecha y retirada del trombo tumoral a través de la incisión realizada en la cava inferior. Extrayendo en bloque hígado derecho con venas suprahepática y suprarrenal derechas incluidas en dicho parche, suprarrenal derecha y trombo.

A 17 pacientes $(60 \%)$ se colocó un parche de Gore-tex ${ }^{\circledR}$ para cubrir el defecto de la vena cava.

\section{Tratamiento complementario}

De los 25 pacientes que presentaron invasión vascular, 14 (56\%) recibieron algún tipo de tratamiento complementario con inmunoterapia, bien con interferón- $\alpha$, Interleukina-II, linfocitos infiltrantes del tumor (células TIL) o células killer activadas por linfokinas (células LAK). El resto de los 11 pacientes (44\%) no preciso tratamiento complementario alguno.

El paciente con el rabdomiosarcoma recibió cinco ciclos de tratamiento poliquimioterápico seguido de radioterapia externa con una dosis de 4500cGy sobre el lecho quirúrgico.

El paciente con el tumor de Wilms siguió tratamiento adyuvante con poliquimioterapia.

\section{Análisis estadístico (sólo en los adenocarcinomas renales)}

Al tratarse de enfermedades distintas, sólo realizamos el análisis descriptivo y de supervivencia en los 25 adenocarcinomas renales. Se realiza un análisis de retrospectivo de los pacientes intervenidos. Para el análisis de la supervivencia, Kaplan-Meyer. Se ha utilizado el paquete informático SPSS- $11^{\circledR}$.

\section{RESULTADOS}

\section{Complicaciones quirúrgicas}

10 pacientes $(35 \%)$ presentaron alguna complicación mayor como consecuencia de la cirugía, incluyendo el fallecimiento. La mortalidad operatoria (considerada desde la cirugía hasta el primer mes post-cirugía) fue de dos pacientes (7\%), uno de ellos intraoperatoriamente por embolia pulmonar masiva como consecuencia de un trombo-embolismo tumoral, y otro al $4^{\circ}$ día postquirúrgico, también por una embolia pulmonar masiva.

Como complicaciones mayores se presentaron tres casos de insuficiencia renal aguda transitoria, uno de ellos precisó hemodiálisis. Y casos aislados de las siguientes complicaciones: una reacción psicótica temporal, como consecuencia de la CEC-H-PC-E, otro caso de pericarditis subclínica, un paciente presentó 
trastornos visuales (hemianopsia transitoria) por la isquemia cerebral, un paciente presentó un cuadro de perforación intestinal iatrogénica (consecuencia del separador ortostático), un caso requirió reintervención por sangrado a las 24 horas de la cirugía, y por último la paciente del feocromocitoma presentó un cuadro de hipokinesia transitoria recuperándose por completo, desconociéndose si fue consecuencia de la cirugía o del fracaso multiorgánico que presentaba previo a la cirugía.

\section{Adenocarcinoma renal}

Al diagnóstico, los pacientes con trombo en cava presentaban una edad media de 54,4 años, una creatinina sérica prequirúrgica: media $1,32 \mathrm{mg} / \mathrm{dl}$. Todos ellos eran varones. Tan sólo un 8\% estaban asintomáticos y un $60 \%$ presentaba más de un signo/sintoma (Tabla 1).

Tabla 1. Estudio descriptivo

\begin{tabular}{|c|c|c|c|}
\hline & & Frecuencia & Porcentaje \\
\hline \multirow{7}{*}{ CLINICA } & Asintomáticos & 2 & $8 \%$ \\
\hline & Dolor Flanco & 3 & $12 \%$ \\
\hline & Masa & 5 & $20 \%$ \\
\hline & Hematuria & 5 & $20 \%$ \\
\hline & Constitucional & 4 & $16 \%$ \\
\hline & Varicocele & 1 & $4 \%$ \\
\hline & Más de uno & 15 & $60 \%$ \\
\hline \multirow[t]{3}{*}{ Estado Actual } & Vivo & 4 & $16 \%$ \\
\hline & Muerto & 19 & $76 \%$ \\
\hline & Perdido & 2 & $8 \%$ \\
\hline \multirow[t]{2}{*}{ Estadio pT } & pT3b2 & 12 & $48 \%$ \\
\hline & pT3c & 13 & $52 \%$ \\
\hline \multirow[t]{3}{*}{ Estadio N } & NO & 16 & $64 \%$ \\
\hline & $\mathrm{N} 1$ & 6 & $24 \%$ \\
\hline & N2 & 3 & $12 \%$ \\
\hline \multirow[t]{2}{*}{ Estadio M } & M0 & 18 & $72 \%$ \\
\hline & M1 & 7 & $28 \%$ \\
\hline \multirow[t]{3}{*}{ Parche cava } & Sí & 17 & $68 \%$ \\
\hline & No & 8 & $32 \%$ \\
\hline & & Media & Mediana \\
\hline \multirow[t]{2}{*}{ Creatinina } & Prequirúrgica & $1,32 \mathrm{mg} / \mathrm{dl}$ & $1,4 \mathrm{mg} / \mathrm{dl}$ \\
\hline & Postquirúrgica & $1,54 \mathrm{mg} / \mathrm{dl}$ & $1,3 \mathrm{mg} / \mathrm{dl}$ \\
\hline \multirow[t]{2}{*}{ Edad } & Media & 54,4 años & \\
\hline & Mediana & 57 años & \\
\hline
\end{tabular}

\section{Estado actual}

De los 25 pacientes tratados, 17 (68\%) han muerto por la enfermedad, $2(8 \%)$ de complicaciones quirúrgicas (1 muerte intraoperatoria), 4(16\%) se encontraban vivos y $2(8 \%)$ se han perdido de seguimiento.
La mediana y media hasta la muerte es de 28,3 y 14,4 meses respectivamente, con un tiempo medio a recidiva de 19,3 meses.

\section{Supervivencia actuarial global}

La media y mediana del seguimiento de todos los pacientes fue 29,7 y 17,7 meses respectivamente. La supervivencia actuarial global es de $29,1 \pm 10 \%$ a tres años y 17,5 $\pm 8 \%$ a cinco años.

\section{Análisis de supervivencia}

Realizando un análisis de supervivencia mediante un Kaplan-Meyer, comparando NOMO vs resto $(\mathrm{N}+\mathrm{MO}, \mathrm{NOM}+, \mathrm{N}+\mathrm{M}+)$ obtenemos unas diferencias estadísticamente significativas a tres y cinco años. De manera que los que no presentan lesiones a distancia (NOMO) tienen una supervivencia a tres años del $50,9 \pm 16,3 \%$ y a cinco años del 38,2 $\pm 16 \%$. Mientras que el resto, es decir, aquellos que presentan algún tipo de lesión a distancia presentan una supervivencia a tres y cinco años de 20,8 $212 \%$ y 10,4 \pm 9 respectivamente (Fig. 2).

\section{OTROS TUMORES}

\section{Tumor de Wilms}

Ocho meses después de la cirugía el paciente permanecía asintomático, posteriormente se perdió el seguimiento.

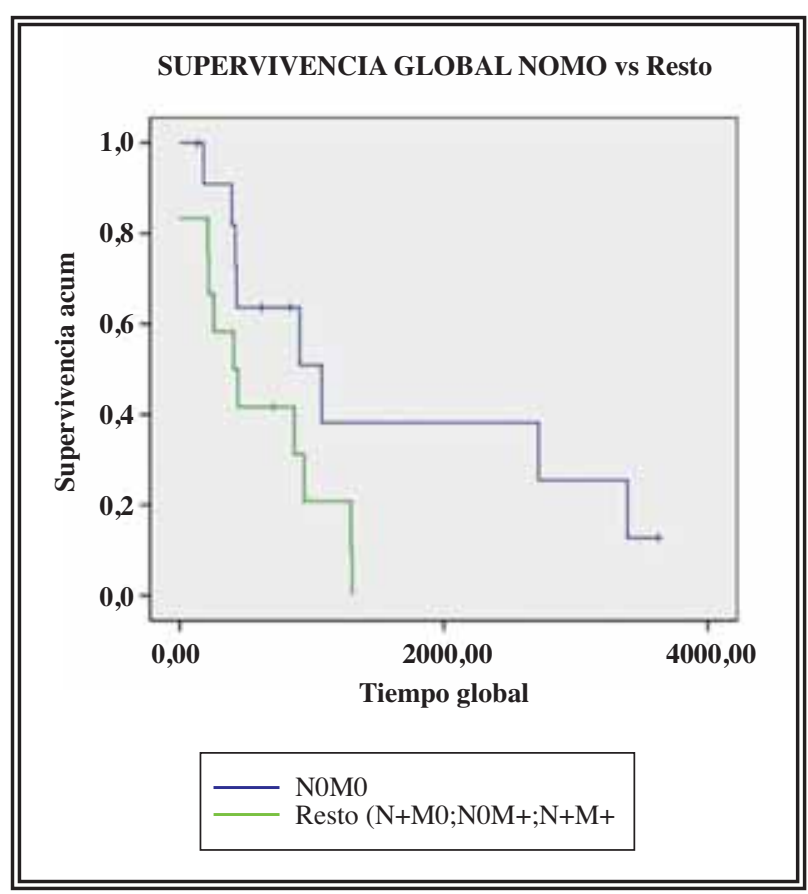

FIGURA 2. Supervivencia global 


\section{Rabdomiosarcoma paratesticular}

Después de 10 años de seguimiento el paciente permanecía asintomático, sin evidencias de extensión tumoral. Posteriormente se perdió el seguimiento.

\section{Feocromocitoma}

En las posteriores revisiones y con un seguimiento superior a 36 meses, la paciente se encuentra asintomática y sin evidencia de enfermedad.

\section{DISCUSIÓN}

El tumor renal con trombo en cava, siempre ha sido una entidad clínico quirúrgica que ha suscitado mucho interés en la comunidad urológica, por su complejidad en el manejo terapéutico, así como en la indicación de su cirugía.

La nefrectomía con trombectomía es el tratamiento de elección en los pacientes con tumor renal y trombo en cava ${ }^{3,24-26}$. Los objetivos de esta cirugía son: incrementar los pacientes potencialmente curables, intentar mejorar la supervivencia de estos pacientes, evitar el tromboembolismo por masa tumoral, controlar y mitigar la sintomatología que estos tumores producen y disminuir la masa tumoral como parte de un tratamiento multimodal ${ }^{22,27-30}$

\section{Nivel del trombo}

En un principio, cuando se publicaron las primeras series, se transmitió que la altura del trombo tenía un significado pronostico, de manera que cuanto más alto era este, peor pronóstico tenía ${ }^{31}$. Sin embargo, más adelante se fueron publicando series con mayor número de pacientes y seguimientos más largos en donde se estableció que la altura del trombo no influía en el pronóstico aunque sí suponía un reto técnico quirúrgico ${ }^{28,32-35}$. En nuestra serie, la presencia de un trombo tumoral no tuvo valor como factor pronostico independiente en la supervivencia global de la enfermedad. Es posible que la elección de la técnica quirúrgica influya en que en algunas series la altura del trombo a su vez influya en la supervivencia. Sin una técnica segura para la cavotomía amplia, puede que la extracción del trombo sea incompleta con los consiguientes riesgos tanto de tromboembolismo como oncológicos.

\section{Factores pronóstico}

Los factores influyentes más significativos en las distintas series, en aquellos pacientes que no presentan lesiones a distancia, son la infiltración de la vía urinaria, la penetración capsular y la extensión a las venas hepáticas, el estatus físico (ECOG; Karnofsky), sintomas locales, caquexia, anemia, recuento de plaquetas ${ }^{28,36-40}$. A su vez factores histológicos como el grado de diferenciación, el subtipo histológico, la presencia de componente sarcomatoide, la invasión microvascular, la necrosis tumoral y la invasión del sistema colector se comportan como factores pronósticos negativos ${ }^{33}$. Toda esta información procede de series de pacientes, habitualmente no muy grandes, por lo que no alcanzan la máxima evidencia científica.

Aunque la presencia de infiltración tumoral de la cava ha sido descrito como peor pronostico por su posibilidad de llevar a márgenes quirúrgicos positivos en ese punto ${ }^{31}$ nosotros, al igual que otros ${ }^{8}$, no hemos podido establecer esta asociación por ser sólo dos los casos con esta infiltración. En ambos casos, gracias a la técnica de exanguinación fue posible la resección completa de la pared afectada y su reconstrucción.

\section{Ganglios positivos}

Sabemos que uno de los factores que más influye en la supervivencia es la presencia de lesiones ganglionares que se comportan como factor independiente en la supervivencia global. Observamos un descenso importante en la supervivencia en aquellos pacientes con lesiones ganglionares y además esta supervivencia fue similar a la de los pacientes con metástasis. Resultados similares a los obtenidos por otros grupos $8,33,41$.

\section{Metástasis}

La presencia de lesiones metastásicas en el momento del diagnóstico supone un escenario muy controvertido. Esa controversia no solo se haya en la indicación de la cirugía, sino también en la interpretación de los resultados de los distintos grupos. Mientras algunos grupos presentan unos resultados de supervivencia a 2 años de un $18 \%^{41}$, otros sin embargo presentan unos resultados más alentadores de hasta un $32 \%+/-13 \%$ a 2 años ${ }^{28}$. En nuestra serie, aquellos pacientes con algún tipo de lesión a distancia presentan una supervivencia a tres y cinco años de $20,8 \pm 12 \%$ y $10,4 \pm 9 \%$ respectivamente. Un argumento a favor de la cirugía radical es como parte de un tratamiento multidisciplinar, para disminuir la carga tumoral previo a un tratamiento complementario y también como parte de un tratamiento paliativo ${ }^{8,29}$. 


\section{Supervivencia}

La supervivencia global observada a tres años $(50,4 \pm 16 \%)$ y cinco años $(38 \pm 16 \%)$ de los pacientes que no tenían metástasis viscerales ni ganglionares sugiere que la invasión tumoral de la vena cava de por sí tiene escasa influencia en la supervivencia. Nuestros resultados están en consonancia con los anteriormente publicados por otros grupos ${ }^{8,34,42,43}$.

\section{Complicaciones}

Uno de los puntos más conflictivos de esta cirugía radical es su tasa morbilidad y mortalidad influidas ambas, en este caso sí, por la altura del trombo. La mortalidad, en series contemporáneas, puede llegar al $12 \%$, las complicaciones mayores al $6 \%$ y las menores al $16 \%^{8,44}$. Nosotros presentamos una mortalidad del 7\% (si se incluyen sólo los renales sube al 8\%). Sin embargo nuestra tasa de complicaciones mayores fue del 27\%. Esta discrepancia la atribuimos a la técnica quirúrgica utilizada. El descenso de la mortalidad quirúrgica puede ser debida a la utilización de CEC-H-PC-E que aunque es una técnica más agresiva que las convencionales, es a su vez más segura, que nos lleva a menor mortalidad por el procedimiento, permitiendo a su vez realizar una trombectomía con mayor seguridad, tanto hemodinámica como oncológica. Por el contrario, esta técnica conlleva una mayor morbilidad asociada, como son las alteraciones de la coagulación, pericarditis, reacciones psicóticas ${ }^{45}$ y otros trastornos neurológicos.

Existen detractores de este tipo de cirugía, tanto por los riesgos neurológicos y hematológicos que conlleva, como por los riesgos oncológicos de diseminación metastática por la realización de CEC-H-PC-E. El potencial de daño neurológico, secundario a la hipotermia y parada cardiocirculatoria, es tiempo dependiente y se pensaba que el intervalo de seguridad era de hasta 45 minutos ${ }^{46,47}$. Sin embargo, estudios más recientes, han demostrado que este intervalo debe ser menor, con límite hasta los 29 minutos a $15^{\circ} \mathrm{C}$. Pasado ese tiempo, la posibilidad del daño neuronal empieza a incrementarse de manera casi exponencial $^{48}$. Por otra parte, la realización de cualquier cirugía con CEC-H-PC-E conlleva un riesgo de coagulopatía del 3\% secundaria a la heparinización necesaria para la realización de la misma ${ }^{49}$.

Respecto al posible riesgo oncológico a causa de la técnica en sí no somos capaces, ni por nuestra experiencia ni por la literatura, contestar a esta cuestión.

\section{Posibles abordajes quirúrgicos}

La altura del trombo tiene importancia a la hora de planificar la cirugía. Varios autores han definido y clasificado el nivel del trombo con este objetivo ${ }^{22,50,51}$. En nuestra opinión, el punto clave es conocer si el trombo tumoral llega a la altura de las venas suprahepáticas.

Cuando el trombo no alcanza las venas suprahepáticas, exponemos la cava en toda la longitud del trombo, clampamos la vena cava y no economizamos la cavotomía para movilizar el trombo con máxima seguridad (resecciones incompletas tras liberación de trombo pueden llevar a embolia pulmonar) En algunos casos el trombo está libre, mientras que en otros se encuentra adherido por fibrina, siendo necesaria su liberalización. En los casos en los que el trombo se encuentra asomando por el ostium de la vena renal (nivel I) se han descrito abordajes laparoscópicos ${ }^{52,53}$, pero el abordaje laparoscopio de trombos más altos está en fase experimental ${ }^{54,55}$.

Si el trombo tumoral alcanza el nivel de las venas suprahepáticas el acceso y el control de la vena cava se hace más difícil ${ }^{44}$. Los problemas a los que hay que enfrentarse son: la disminución del retorno venoso tras el clampaje de cava que puede llevar a una hipotensión profunda; sangrado durante la cavotomía procedente de las vevas suprarenales no controladas y la producción de congestión hepática. Algunas técnicas se han descrito para intentar evitar estas dificultades como el bypass veno-veno entre la cava distal y la aurícula derecha y así proporcionar al corazón el volumen sanguíneo necesario ${ }^{56}$. El sangrado procedente de las venas suprahepáticas se intenta controlar mediante varias maniobras como el clampaje de la arteria hepática y de la vena porta (maniobra de Pringle). Estas maniobras tienen un tiempo muy limitado ya que durante este el hígado se somete a isquemia caliente y se aumenta el riesgo quirúrgico.

Por ello, en los casos con trombo que alcanzan las venas suprahepáticas hasta la aurícula, indicamos CEC-H-PC-E. Marshall et al. ${ }^{21}$ y Krane et al. ${ }^{57}$ en 1984, basándose en técnicas de cirugía cardiaca infantil para el tratamiento de malformaciones congénitas, fueron los pioneros de CEC-H-PC-E en tumores con trombo a la altura de la vena cavavenas suprahepáticas o por encima (tumores T3B2T3C). Posteriormente se difundió con gran éxito, por numerosos autores en el campo de la urología para el tratamiento de tumores renales y otros tumores retroperitoneales con invasión venosa ${ }^{16,17,58-61}$. 
Nosotros, en los casos con trombo que alcanzan las venas suprahepáticas o hasta la aurícula, indicamos CEC-H-PC-E junto con la cirugía de resección del tumor abdominal. Precisa siempre de la colaboración de cirujanos cardiacos, ocasionalmente de cirujanos hepáticos y obliga a una gran implicación de los anestesistas.

Otros grupos prefieren realizar técnicas similares pero menos agresivas, como son la realización de CEC-H-PC-E, pero a través de una miniesternotomía sobre la cuarta costilla, evitando los riesgos de una esterenotomía media tradicional, pero ofreciendo seguridad frente a sangrados masivos. Descrita por Cosgrove y Sabik ${ }^{62}$ para el tratamiento de lesiones valvulares y aplicada en el tratamiento de tumores renales por Fitzgerald et $\mathrm{al}^{63}$. Recientemente se ha publicado un estudio en donde se compara la técnica con la tradicional esternotomía media con la variante descrita de mini toracotomía, mejorando los tiempos quirúrgicos y la estancia hospitalaria pero con similares resultados quirúrgicos y oncológicos ${ }^{64}$.

El rabdomiosarcoma retroperitoneal presentado es el único publicado con trombo en vena cava y larga supervivencia ${ }^{17}$ y en el caso del tumor de Wilms encontramos en la literatura sólo casos aisla$\operatorname{dos}^{11,16}$. El feocromocitoma raramente se extiende dentro de la vena cava. Hartgrink y cols ${ }^{65}$ en una revisión realizada en 2001 encuentran unos 20 casos y sólo 3 con extensión hasta la aurícula derecha, tratándose en dos casos de feocromocitomas resecados previamente, encontrado un único caso de feocromocitoma primario con afectación de la vena cava y aurícula derecha.

Podemos concluir, y coincidiendo con la literatura, que los tumores renales con trombo en cava tiene mal pronóstico por la alta asociación de metástasis ganglionares y a distancia y el tamaño tumoral. El pronóstico es independiente de la longitud del trombo. La utilización de técnicas quirúrgicas con CEC-H-PC-E, esta justificada y su utilización no empeora la supervivencia.

La utilización de esta técnica en otras patologías tumorales que se asocian a trombo en vena cava, está indicada por sus resultados, permitiendo una resección tumoral de una manera segura y reproductible. En nuestra opinión sólo se incrementa la supervivencia de estos pacientes con opciones quirúrgicas que asocien seguridad quirúrgica con resecabilidad completa.

\section{REFERENCIAS}

1. García OD, Fernández Fernández E, de Vicente E, Honrubia A, Moya JL, Abella V, et al. Estratégia quirúrgica en el carcinoma de células renales con extensión a la circulación venosa. Actas Urol Esp. 2005;29(5):448-456.

2. Skinner DG, Colvin RB, Vermillion CD, Pfister RC, Leadbetter WF. Diagnosis and management of renal cell carcinoma. A clinical and pathologic study of 309 cases. Cancer. 1971;28(5): 1165-1177.

3. Marshall FF, Dietrick DD, Baumgartner WA, Reitz BA. Surgical management of renal cell carcinoma with intracaval neoplastic extension above the hepatic veins. J Urol. 1988;139(6):11661172 .

4. Bastian PJ, Haferkamp A, Akbarov I, Albers P, Müller SC. Surgical outcome following radical nephrectomy in cases with inferior vena cava tumour thrombus extension. Eur J Surg Oncol. 2005;31(4):420-423. Epub 2005.

5. NCCN practice guidelines for kidney cancer. National Comprehensive Cancer Network. Oncology (Williston Park). 1998;12 (11A):396-412.

6. Cherrie RJ, Goldman DG, Lindner A, deKernion JB Prognostic implications of vena caval extension of renal cell carcinoma. J Urol. 1982;128(5):910-912.

7. Kuczyk M, Hofner K, Stief C et al. Surgical strategy in late-stage renal cell cancer with intracaval extension. Curr Opin Urol. 1998;8(3):241-245.

8. Parekh DJ, Cookson MS, Chapman W, Harrell F Jr, Wells N, Chang SS, et al. Renal cell carcinoma with renal vein and inferior vena caval involvement: clinicopathological features, surgical techniques and outcomes. J Urol. 2005;173(6):1897-1902.

9. Montie JE, Jackson CL, Cosgrove DM, Streem SB, Novick AC, Pontes JE. Resection of large inferior vena caval thrombi from renal cell carcinoma with the use of circulatory arrest. J Urol. 1988;139(1):25-28.

10. Libertino JA, Zinman L, Watkins E Jr. Long-term results of resection of renal cell cancer with extension into the inferior vena cava. J Urol. 1987;137(1):21-24.

11. De Castro F, Robles JE, Zudaire JJ et al.: Tratamiento quirúrgico de la trombosis tumoral de la vena cava inferior. Rev Cir Ibero-Am, 1: 254, 1992.

12. Matthews PN, Evans C, Breckenridge IM. Involvement of the inferior vena cava by renal tumour: surgical excision using hypothermic circulatory arrest. BJU Int. 1995;75(4):441-444.

13. Menke, J, Grabbe E. Metastatic Germ-Cell Cancer. N Engl J Med. 2007;357(4):391.

14. Aguera Fernández L, Zudaire Bergera JJ, Llorens León R, Rosell Costa D, De Castro Barbosa F, Isa Kroon W, et al. By pass cardiopulmonar con exanguinotransfusión e hipotermia profunda en el tratamiento quirúrgico del trombo tumoral en la vena cava causado por el carcinoma renal. Actas Urol Esp. 1990;14(5):339-340.

15. De Castro F, Rosell Costa D, Zudaire Bergera J et al. Tratamiento quirúrgico del trombo en vena cava con circulación extracorpórea, exanguinotransfusión e hipotermia profunda. Rev Cir Ibero Am. 1: 254, 1992.

16. Rodríguez-Rubio FI, Abad JI, Sanz G, Diez-Caballero F, MartínMarquina A, Rosell D et al. Surgical management of retroperitoneal tumors with vena caval thrombus in the inferior cava using cardiopulmonary bypass, arrested circulation and profound hypothermia. Eur Urol. 1997;32(2):194-197.

17. Rodríguez-Rubio FI, Abad JI, Rábago G, Berián JM. Long survival of a patient with paratesticular rhabdomiosarcoma inferior vena caval involvement. Urology. 1997;50(6):978-979. 
18. López Ferrandis J, Saiz Sansi A, Cervera Delgado M, Rioja Zuazu J, Regojo Balboa JM, Berián Polo JM. Pheocromocitoma with thrombus in cava. Actas Urol Esp. 2005;29(3):281-286.

19. De Castro F, Agüera LG, Robles JE, Llorens R, Abad JI, Rosell $\mathrm{D}$, et al. Leimiosarcoma de la vena cava: Presentación de dos casos clínicos. Actas Urol Esp. 1993;17(8):504-507.

20. Rioja Zuazu J, Zudaire Bergera J, Rincón Mayans A et al. Carcinoma renal con trombo en cava: Resultados. In: Libro de abstracts LXXI Congreso Nacional de Urología. Sevilla, 2006.

21. Marshall FF, Reitz BA, Diamond DA. A new technique for management of renal cell carcinoma involving the right atrium: hypothermia and cardiac arrest. J Urol. 1984;131(1):103-107.

22. Neves RJ, Zincke H. Surgical treatment of renal cancer with vena cava extension. Br J Urol. 1987;59(5):390-395.

23. UICC: TNM Classification of Malignant Tumours. New York: Wiley.Liss, 2002.

24. Kaplan S, Ekici S, Do€an R, Demircin M, Ozen H, Paflao€lu I. Surgical management of renal cell carcinoma with inferior vena cava tumor thrombus. Am J Surg. 2002;183(3):292-299.

25. Swierzewski DJ, Swierzewski MJ, Libertino JA. Raddicla Nephrectomy in patients with renal cell carcinoma with venous, vena caval, and atrial extension. Am J Surg. 1994;168(2):205209.

26. Belis JA, Levinson ME, Pae WE Jr. Complete radical nephrectomy and vena caval thrombectomy during circulatory arrest. J Urol. 2000;163(2):434-436.

27. Skinner DG, Pfister RF, Colvin R. Extension of renal cell carcinoma into the vena cava: the rationale for aggressive surgical management. J Urol. 1972;107(5):711-716.

28. Zisman A, Wieder JA, Pantuck AJ, Chao DH, Dorey F, Said JW, et al. Renal cell carcinoma with tumor thrombus extension: biology, role of nephrectomy and response to immunotherapy. J Urol, 2003;169(3):909-916.

29. Hatcher PA, Anderson EE, Paulson DF, Carson CC, Robertson JE. Surgical management and prognosis of renal cell carcinoma invading the vena cava. J Urol. 1991;145(1):20-23; discussion 23-24.

30. Glazer AA, Novick AC. Long-term followup after surgical treatment for renal cell carcinoma extending into the right atrium. J Urol. 1996;155(2):448-450.

31. Sosa RE, Muecke EC, Vaughan ED Jr, McCarron JP Jr. Renal cell carcinoma extending into the inferior vena cava: the prognostic significance of the level of vena caval involvement. J Urol. 1984;132(6): 1097-100.

32. Libertino JA, Zinman L, Watkins E Jr. Long-term results of resection of renal cell cancer with extension into inferior vena cava. J Urol. 1987;137(1):21-24.

33. Sweeney P, Wood CG, Pisters LL, Slaton JW, Vaporciyan A, Munsell M, et al. Surgical management of renal cell carcinoma associated with complex inferior vena caval thrombi. Urol Oncol. 2003;21(5):327-333.

34. Bissada NK, Yakout HH, Babanouri A, Elsalamony T, Fahmy W, Gunham M et al. Long-term experience with management of renal cell carcinoma involving the inferior vena cava. Urology. 2003;61(1):89-92

35. Tsuji Y, Goto A, Hara I, Ataka K, Yamashita C, Okita et al. Renal cell carcinoma with extension of tumor thrombus into the vena cava: surgical strategy and prognosis. J Vasc Surg. 2001;33(4):789-796

36. Kim HL, Belldegrun AS, Freitas DG, Bui MH, Han KR, Dorey FJ, et al. Paraneoplastic signs and symptoms of renal cell carcinoma: implications for prognosis. J Urol. 2003;170(5):17426174.
37. Kim HL, Han KR, Zisman A, Figlin RA, Belldegrun AS. Cachexia-like symptoms predict a worse prognosis in localized t1 renal cell carcinoma. J Urol. 2004;171(5):1810-1813.

38. Patard JJ, Leray E, Cindolo L, Ficarra V, Rodriguez A, De La Taille A, et al. Multi-institutional validation of a symptom based classification for renal cell carcinoma. J Urol. 2004; 172(3):858-862.

39. Bensalah K, Leray E, Fergelot P, Rioux-Leclercq N, Tostain J, Guillé $\mathrm{F}$, et al. Prognostic value of thrombocytosis in renal cell carcinoma. J Urol. 2006;175(3 Pt 1):859-683.

40. Patard JJ, Leray E, Rodriguez A, Rioux-Leclercq N, Guillé F, Lobel B. Correlation between symptom graduation, tumor characteristics and survival in renal cell carcinoma. Eur Urol. 2003;44(2):226-232.

41. Staehler G, Brkovic D. The role of radical surgery for renal cell carcinoma with extension into the cava. J Urol. 2000;163(6): 1671-1675.

42. Montie JE, el Ammar R, Pontes JE, Medendorp SV, Novick AC, Streem SB, et al. Renal cell carcinoma with inferior vena cava tumor thrombi. Surg Gynecol Obstet. 1991;173(2):107-115.

43. Skinner DG, Pritchett TR, Lieskovsky G, Boyd SD, Stiles QR. Vena caval involvement by renal cell carcinoma. Surgical resection provides meaningful long-term survival. Ann Surg. 1989;210(3):387-392; discussion 392-394

44. Vaidya A, Ciancio G, Soloway M. Surgical techniques for treating a renal neoplasm invading the inferior vena cava. J Urol. 2003;169(2):435-444.

45. Sockalingam S, Parekh N, Bogoch II, Sun J, Mahtani R, Beach $\mathrm{C}$, et al. Delirium in the postoperative cardiac patient: a review. J Card Surg. 2005;20(6):560-567.

46. Ergin MA, Griepp EB, Lansman SL, Galla JD, Levy M, Griepp RB. Hypothermic circulatory arrest and other methods of cerebral protection during operations on the thoracic aorta. J Card Surg. 1994;9(5):525-537.

47. Ergin MA, Phillips RA, Galla JD, Lansman SL, Mendelson DS, Quintana CS, et al. Significance of distal false lumen after type A dissection repair. Ann Thorac Surg. 1994;57(4):820-824; discussion 825 .

48. McCullough JN, Zhang N, Reich DL, Juvonen TS, Klein JJ, Spielvogel D, et al. Cerebral metabolic suppression during hypothermic circulatory arrest in humans. Ann Thorac Surg. 1999;67(6):1895-1899; discussion 1919-1921.

49. Papadimitriou DK, Pitoulias GA, Tachtsi MD, Koutsias SG, Radopoulos DK. Surgical treatment of renal neoplasmatic thrombi extending into the inferior vena cava. Ann Vasc Surg. 2006;20(2):223-227.

50. Wilkinson C, Kimovec M, Uejima T. Cardiopulmonar bypass in patients with malignant renal neoplasm. Br J Anaesth, 58: $461,1986$.

51. Libertino JA. Renal cancer with extension into the vena cava. London: Butterworths, p. 127, 1986.

52. Savage SJ, Gill IS. Laparoscopic radical nephrectomy for renal cell carcinoma in a patient with level I renal vein tumor thrombus. J Urol. 2000;163(4):1243-1234.

53. Disanto V, Pansadoro V, Portoghese F, Scalese GA, Romano M. Retroperitoneal laparoscopic radical nephrectomy for renal cell carcinoma with infrahepatic vena caval thrombus. Eur Urol. 2005;47(3):352-356.

54. Fergany AF, Gill IS, Schweizer DK, Kaouk JH, ElFettouh HA, Cherullo EE et al. Laparoscopic radical nephrectomy with level II vena caval thrombectomy: survival porcine study. J Urol. 2002;168(6):2629-2631.

55. El-Galley R. Laparoscopic right nephrectomy using a novel technique to excise an inferior vena-caval cuff in a porcine model. J Endourol. 2005;19(8):1032-1035. 
56. Burt, M. Inferior vena caval involvement: Use of venovenous by-pass adjunct during resection. Urol Clin North Am. 18: 437, 1991.

57. Krane RJ, deVere White R, Davis Z, Sterling R, Dobnik DB, McCormick JR. Removal of renal cell carcinoma extending into the right atrium using cardiopulmonary bypass, profound hypothermia and circulatory arrest. J Urol. 1984;131(5):945947.

58. De Castro Barbosa F, Robles Garcia J, Rosell Costa D et al. Tumor thrombusis in inferior vena cava: Diagnostic imagin and therapeutical approximation. Actas Urol Esp, 16: 373, 1992.

59. Gonzalez Martin, M., Chantada Abal, V., Alvarez Castelo, L. et al.: Carcinoma Renal con trombo tumoral en vena cava y aurícula. Experiencia y Revisión. Arch Esp Urol, 51: 44, 1998.

60. Padilla Nieva J, Lekumberri Castaños D, Crespo Antin V et al. Renal carcinoma with ven cava invasion. A new case. Actas Urol Esp, 25: 125, 2001.

61. Kuczyk MA, Münch T, Machtens S, Grünewald V, Jonas U. The impact of extracorporal circulation on therapy-related mortality and long-term survival of patients with renal cell cancer and intracaval neoplastic extension. World J Urol. 2002;20(4):227231.
62. Cosgrove DM 3rd, Sabik JF. Minimally invasive approach for aortic valve operations. Ann Thorac Surg, 1996;62(2):596-597.

63. Fitzgerald JM, Tripathy U, Svensson LG, Libertino JA. Radical nephrectomy with vena caval thrombectomy using a minimal access for caridopulmonar bypass. J Urol. 1998;159(4):12921293.

64. Wotkowicz C, Libertino JA, Sorcini A, Mourtzinos A. Management of renal cell carcinoma with vena cava and atrial thrombus: minimal access vs median sternotomy with circulatory arrest. BJU Int. 2006;98(2):289-297.

65. Hartgrink HH, Roelfsema F, Tollenaar RA, Hiddema PA, Pij1 $\mathrm{ME}$, van de Velde CJ. Primary pheochromocytoma extending into the right atrium: report of a case and review of the literature. Eur J Surg Oncol. 2001;27(1):115-119.

Correspondencia autor: Dr. J. Rioja Zuazu

Dept. Urology G4. Academisch Medisch Centrum

Meibergdreef 9 - 1105 AZ Amsterdam The Netherlands Holanda Tel.: +31 205669111

E-mail autor: jriojazu@gmail.nl

Información artículo: Original - Tumor renal

Trabajo recibido: enero 2008

Trabajo aceptado: febrero 2008 